$30 / 6 / 11$

\title{
Comparison of small ELMs on MAST and ASDEX Upgrade
}

\author{
A. Kirk, H.W. Muller ${ }^{\mathrm{a}}$, E. Wolfrum ${ }^{\mathrm{a}}$, H. Meyer, A. Herrmann ${ }^{\mathrm{a}}$, T. Lunt ${ }^{\mathrm{a}}$, V. Rohde ${ }^{\mathrm{a}}$, \\ P. Tamain ${ }^{\mathrm{b}}$ and the MAST and ASDEX Upgrade Team
}

EURATOM/CCFE Fusion Association, Culham Science Centre, Abingdon, Oxon OX14 3DB, UK

${ }^{a}$ Max-Planck Institut für Plasmaphysik, EURATOM Association, Garching, Germany

${ }^{b}$ Association Euratom-CEA, CEA/DSM/IRFM, CEA-Cadarache, F-13108 St Paul-lezDurance Cedex, France

\begin{abstract}
Results from a set of ITPA-coordinated experiments on ASDEX Upgrade and MAST to compare the characteristics of small edge-localized modes (ELMs) are presented. In MAST a small ELM regime is established in connected double null discharges in a limited region of normalised pedestal pressure and collisionality. Type II ELMs on ASDEX Upgrade have high frequency and low energy loss and occur at high triangularity and close to double null. On both devices the transition from type I to small ELMs is connected with a similar threshold value of the pedestal collisionality. For the first time the temporal and spatial evolution of the filament structures observed during these small ELMs has been studied. The radial and toroidal velocities of the filaments in these small ELMs are slower compared to type I ELMs on both devices. The observations are compatible with the filaments in small ELMs originating closer to the last closed flux surface than is the case in type I ELMs. The toroidal mode number of the small ELMs, derived from the temporal evolution of the filaments, is typically a factor of two larger than for type I ELMs. The small ELMs on MAST have sufficient similarities to type II ELMs on ASDEX Upgrade that they should be classified as the same.
\end{abstract}




\section{Introduction}

The performance of future devices, such as ITER, relies on H-mode operation with high pedestal pressures. The resulting high pressure gradients at the edge of the plasma result in plasma instabilities called Edge Localised Modes (ELMs), which periodically eject particles and energy from the plasma core towards the in-vessel components. One confusing issue associated with ELMs is that there are a (growing) number of different 'types' into which ELMs are categorized according their experimental characteristics [1][2]. The type that produces the largest energy loss from the plasma, type I, are predicted to be too large to be regularly tolerated in ITER, because of the damage they would cause to in vessel components [3]. Accordingly, there is a search for good confinement regimes which have no or small ELMs. Small ELM regimes have been observed on a wide range of machines (see [1] and references therein), most of these however are not produced in plasmas with an ITER relevant edge (mainly because the edge collisionality is too high). Several ELM regimes exist which have a small energy loss (see [4] and references therein), one of which is the type II ELM regime, which has been observed at high pedestal collisionalities $\left(v_{\mathrm{e}}^{*}\right)[5]$ on ASDEX Upgrade [6], JET [7] and other devices. The advantage of this regime is that the global energy confinement can be as high as in a type I ELMing regimes whilst the energy loss per ELM is small [2]. A stability analysis carried out on the ASDEX Upgrade data using the ELITE code shows that when compared to type I ELMs, type II ELMs have a narrower radial eigenfunction of the unstable peeling-ballooning mode [8]. A stability analysis carried out on the JET data concluded that in type II ELMy 
plasmas the intermediate-n peeling modes are avoided due to the closeness to double null and type II ELMs are triggered by high-n ballooning modes [9]. A characteristic feature of type II ELM regimes in several machines is the appearance of fluctuations in the range of $10-60 \mathrm{kHz}$ [10]. The fluctuations move in the electron drift direction and lead to a small reduction of the edge electron temperature gradients. The reduction in edge pressure gradients is connected with these MHD fluctuations, which affect only electron temperatures but not electron densities [1].

A similar small ELM regime has been observed at $v_{\mathrm{e}}^{*}>1$ on MAST [12] but to date it has not been possible to identify whether these are the same as type II ELMs or not. A previous ITPA-coordinated experiment between Alcator C-Mod, MAST and NSTX compared the characteristics and access conditions to small ELM regimes on these devices [13]. The goal of the ITPA-co-ordinated experiment described in this current paper, which was performed on ASDEX Upgrade and MAST, was to measure the properties of type II ELMs on ASDEX Upgrade and compare them to the small ELMs observed on MAST to see if they could be identified as the same type. Although type II ELMs are probably not obtainable in ITER, because the ITER pedestal will have a much lower collisionality $v_{\mathrm{e}}^{*}<0.1$, a study of their properties may help to understand why type II ELMs exist and why their energy loss is so small, which may open a way to establish other ITER relevant regimes. In addition while the spatial structure of type I ELMs has been studied in detail (see [14] and references therein), very little work has been done on the study of type II ELMs. 


\section{Existence space for type II ELMs on AUG and small ELMs on MAST}

Type II ELMs are typically observed in ASDEX Upgrade in high triangularity (upper triangularity $\delta_{\mathrm{u}}=0.33$, lower triangularity $\delta_{\mathrm{l}}=0.45$ ) discharges operated near to a Connected Double Null $(\mathrm{CDN})$ configuration. In the discharges presented here, the plasma current $\left(\mathrm{I}_{\mathrm{P}}\right)$ was $800 \mathrm{kA}$, with a magnetic field on axis $\left(\mathrm{B}_{\mathrm{T}}\right)$ of $2.5 \mathrm{~T}$ and an edge safety factor $\left(\mathrm{q}_{95}\right)$ of 5.5. Further details on these discharges can be found in reference [1]. The plasma started off in a lower Single Null Diverted (SND) configuration with the ion $\nabla$ B drift direction towards the lower targets and the $\mathrm{Z}$ position of the plasma was scanned upwards so changing the distance between the two separatrices at the outboard mid-plane $\left(\delta r_{\text {sep }}\right)$ from a negative number (lower SND) to a positive number (upper SND). The effect of this scan on the ELM behaviour can seen in Figure 1a, which shows time traces of the divertor $\mathrm{D}_{\alpha}$ and $\delta r_{\text {sep }}$. There are three distinct phases: for $\mathrm{t}<2.9 \mathrm{~s}$ when $\delta \mathrm{r}_{\text {sep }}<-0.7 \mathrm{~cm}$ type I ELMs are present, in the period $3.2<\mathrm{t}<3.5 \mathrm{~s}$, when $-0.5<\delta \mathrm{r}_{\text {sep }}<0.1 \mathrm{~cm}$ type II ELMs are present and finally for $\mathrm{t}>3.5 \mathrm{~s}$ when $\delta \mathrm{r}_{\text {sep }}>0.1 \mathrm{~cm}$ type I ELMs return. In subsequent discharges the $\mathrm{z}$-shift of the plasma is stopped at $\mathrm{t}=2.9 \mathrm{~s}\left(\delta \mathrm{r}_{\mathrm{sep}}=-0.5 \mathrm{~cm}\right)$ and the type II ELM period extends for the remainder of the flat top period (Figure 1b). This $\delta r_{\text {sep }}$ scan allows the characteristics of the type I and type II ELMs to be investigated in the same shot with similar plasma parameters.

In MAST small ELMs are observed in CDN discharges $\left|\delta \mathrm{r}_{\text {sep }}\right|<0.2 \mathrm{~cm}$ with $\mathrm{I}_{\mathrm{p}}=0.7$ MA, $\mathrm{B}_{\mathrm{T}}=0.5 \mathrm{~T}, \mathrm{q}_{95}=5.5, \kappa=1.9$, and $\delta=0.43$. A power scan was conducted to determine the operational window for small ELMs . Figure 2 shows time traces of the divertor $\mathrm{D}_{\alpha}$ emission for three of the discharges in the power scan. In the lower power discharges type 
III ELMs were observed (Figure 2a). As the power is increased, after a short type III ELMing period extending to $0.31 \mathrm{~s}$ and before the first type I ELM at $0.36 \mathrm{~s}$, small ELMs can be observed (see the small oscillations in the $\mathrm{D}_{\alpha}$ signals between 0.32 and $0.36 \mathrm{~s}$ in Figure 2b). As the beam power is increased further the type I ELMing regime is established earlier and the small ELMs disappear (Figure 2c). For intermediate beam powers between 1.3 and 1.7 MW the small ELMs can co-exist with type III ELMs at the low end and type I ELMs at the upper end, but there is a clear threshold below and above which they no longer exist. The collisionality is calculated following reference [5] as:

$$
v_{e}^{*}=6.921 .10^{-18} \frac{\operatorname{Rqn}_{e} Z_{e f f} \ln \Lambda_{e}}{\varepsilon^{3 / 2} T_{e}^{2}}
$$

where $\mathrm{R}$ is the major radius in $\mathrm{m}, \mathrm{q}_{95}$ is the safety factor at $95 \%$ of flux surface and where $\varepsilon$ is the inverse aspect ratio. $Z_{\text {eff }}$ is the effective ion charge, $n_{e}$ the electron density in $\mathrm{m}^{-3}$ and $T_{e}$ the temperature in $\mathrm{eV}$ evaluated at the top of the pedestal. $\ln \Lambda_{\mathrm{e}}$ is the Coulomb logarithm defined by $\ln \Lambda_{e}=31.3-\ln \left(\sqrt{n_{e}} / T_{e}\right)$. Figure 3a shows that there is a wide range in normalised pedestal pressure $\left(\beta_{\mathrm{ped}}\right)$ and pedestal top collisionality $\left(v_{\mathrm{e}}\right)$ space for which the small ELMs occur with $1.5<v_{\mathrm{e}}{ }^{*}<20$, and an upper limit of $\beta_{\text {ped }} \sim 4 \%$. In lower SND discharges no small ELMs have been observed.

In ASDEX Upgrade the discharges were performed with a range of neutral beam heating power and gas fuelling rates to determine the existence space for type II ELMs. As can be seen from Figure 3b, relative to type III and type I ELMs the type II ELMs on ASDEX Upgrade occupy a similar region of the $\beta_{\text {ped }}$ versus $v^{*}$ e space as the small ELMs on MAST. While the location of the small ELMs relative to the other ELM types is the same 
on the two devices there is not a unique window in the value of either $\beta_{\text {ped }}$ or $v_{\mathrm{e}}^{*}$. A similar observation was made for experiments between Alcator C-Mod, NSTX and MAST where is was found that, although the access condition for small ELMs occurs in apparent $\beta_{\text {ped }}$ windows, the lower and upper boundaries of the windows differ between devices [13].

\section{The spatial and temporal structure of small ELMs on MAST}

The filamentary nature of ELMs has been previously studied on MAST using high speed visible imaging of the plasma boundary [15]. In the experiments described in this paper, a Photon Ultima APX-RS was used to continuously record unfiltered light, dominated by $\mathrm{D}_{\alpha}$ emission, from a reduced view centred on the mid-plane Low Field Side (LFS) portion of the plasma at a frame rate of $100 \mathrm{kHz}$. Figure 4 shows a single frame captured during a small ELM. The image shows a strip of the plasma approximately $20 \mathrm{~cm}$ high. The left hand side of the image shows the Last Closed Flux Surface (LCFS) at the LFS of the plasma. The High Field Side (HFS) LCFS is located out of the image at the right and side (for further details of the view see figure 2 in reference [16]). A regular set of bands, representing the filamentary structures are observed. These small ELMs have a high number of filaments, representing a high toroidal mode number, with about twice as many filaments being observed than in a typical type I ELM [15].

The propagation of the filaments observed during each ELM has been measured by determining the toroidal and radial location of each filament in subsequent frames, separated by $10 \mu$ s. Figure 5 shows a set of frames obtained during a single small ELM; the time of each frame relative to the $\mathrm{D}_{\alpha}$ emission intensity is shown in Figure $5 \mathrm{a}$ and the ion saturation current $\left(\mathrm{I}_{\mathrm{SAT}}\right)$ measured by a reciprocating probe located at the outboard mid- 
plane at a distance of $5 \mathrm{~cm}$ from the LCFS is shown in Figure $5 \mathrm{~b}$. The images have been analysed by mapping 3-D field lines, generated from the magnetic equilibrium at various distances outside the LCFS, onto the 2-D image [15]. The toroidal angle of these projected field lines is then modified so as to minimise the difference between the mapped field line and the observed filament. The dotted blue curve in frame 6 represents a filament that is located at the LCFS, whilst the solid red curve in frames 4 to 7 represents a filament that is propagating radially outwards. The fact that some of these filaments do travel radially can be seen from the peaks in the $\mathrm{I}_{\mathrm{SAT}}$ signal shown in Figure $5 \mathrm{~b}$ which represent the interaction of filaments with the probe. The light intensity associated with these small ELM filaments is much lower than that associated with filaments observed in type I ELMs [16] suggesting that they carry a relatively small density.

Although some of the filaments separate from the LCFS during the ELM the majority appear to remain close to the LCFS. Therefore, in addition to the manual tracking described above, a semi-automated mode analysis has been used, in which the filament location is fixed at the location of the LCFS. The field lines are then mapped onto the image and the intensity along the field line calculated as a function of toroidal angle. A peak finding detection algorithm is then applied to the trace of intensity versus toroidal angle (Figure 6) and results in the toroidal location (vertical lines) and the half width half maximum (HWHM) toroidal extent (horizontal line) of the filaments being determined. In the example shown in Figure 6, (which is the result of the analysis of frame 2 of Figure 5) 6 regularly spaced filaments are identified. The mean separation in toroidal angle is $13^{\circ}$, corresponding to a toroidal mode number of $\sim 28$. The mean toroidal size of each filament 
(HWHM) is $2.6^{\circ}$ corresponding to $\sim 6 \mathrm{~cm}$. The same technique is then applied to subsequent frames to determine the toroidal propagation of each filament (Figure 7 shows the results of the analysis applied to the frames shown in Figure 5). A single filament can exist for up to $140 \mu \mathrm{s}$. Each of the 9 filaments identified in Figure 7 is observed to rotate with approximately constant velocity moving $\sim 2^{\circ}$ every $10 \mu$ s corresponding to a toroidal velocity $\left(\mathrm{V}_{\phi}\right)$ of $\sim 5 \mathrm{kms}^{-1}$ in the same direction as the plasma rotation. The frame to frame location of each filament can be determined with an accuracy of $0.5^{\circ}$, corresponding to an uncertainty in the velocity of $1.25 \mathrm{kms}^{-1}$. For comparison the toroidal rotation velocity of the pedestal in this discharge, which is measured using charge exchange recombination spectroscopy is $\sim 15 \mathrm{kms}^{-1}$. It is not possible to tell from measurements made on the images whether the rotation of the filaments is purely toroidal, poloidal or some mixture of the two. In this paper all rotations will be expressed as though the rotation is only in the toroidal direction.

Measurements of the separation and toroidal propagation of the filaments while they remain at the LCFS have been repeated for all the ELMs in a series of shots with increasing neutral beam energy. Figure 8 a shows the probability distribution function for the toroidal velocity $\left(\mathrm{V}_{\phi}\right)$ for type I, III and small ELMs. The number of filaments tracked is 64,93 and 189 for the type I, II and III ELMs respectively. In type I and III ELMs the filaments start off rotating at a constant toroidal velocity but decelerate toroidally before they move radially outwards [15], therefore the toroidal velocity plotted in Figure 8a is that obtained during the initial stage when the toroidal velocity is constant. 
Type I ELMs have the largest values of $\mathrm{V}_{\phi}$ with a mean of $13.9 \mathrm{kms}^{-1}$ and standard deviation $(\sigma)$ of $2.9 \mathrm{kms}^{-1}$, which is comparable to the toroidal rotation velocity of the plasma at the pedestal top $\left(\Psi_{\mathrm{N}}=0.95\right) \mathrm{V}_{\phi}^{\text {ped }}=15 \mathrm{kms}^{-1}$. The type III ELMs have a slightly lower $\mathrm{V}_{\phi}\left(\right.$ mean $\left.=11.0 \mathrm{kms}^{-1} \sigma=3.2 \mathrm{kms}^{-1}\right)$, but this is again comparable to the rotation velocity at the top of the pedestal in these shots which varies between 8 and $15 \mathrm{kms}^{-1}$ and has $\left\langle\mathrm{V}_{\phi}^{\text {ped }}\right\rangle=12 \mathrm{kms}^{-1}$ and is smaller due to the lower input beam power in the type III ELM-ing discharges. The small ELMs have lower $\mathrm{V}_{\phi}\left(\right.$ mean $=5.6 \mathrm{kms}^{-1} \mathrm{\sigma}=1.1 \mathrm{kms}^{-}$

${ }^{1}$ ), much smaller than the toroidal velocity at the top of the pedestal, which is similar to that in the type I ELM-ing part of the discharge $\left(\left\langle\mathrm{V}_{\phi}{ }^{\text {ped }}>=15 \mathrm{kms}^{-1}\right)\right.$. In order for these filaments to rotate with the plasma they would have to originate from a location further out from the pedestal top where the rotation velocity is lower, presumably in the steep sheer region of the pedestal velocity profile. Figure $8 \mathrm{~b}$ shows the derived toroidal mode number (n) of the various ELM types. The type I ELMs have the lowest value of $n$ with a mean of 12, the small ELMs have the largest toroidal mode number with a mean value of 25 . The type III ELMs cover a large range from $n=5$ to 30 .

The radial positions of the filaments that leave the LCFS have also been tracked as a function of time. The number of filaments tracked is 22,15 and 28 for type I, II and III ELMs respectively. As has been reported previously the type I ELM filaments accelerate away from the edge i.e. at least over the first few frames after they separate from the LCFS the change of radial distance detected from frame to frame increases [15]. However, for the case of the filaments observed in the small ELMs the change in radial location from one frame to another is the same i.e. consistent with them having a constant radial velocity. 
Figure 9a shows an example of the radial location of a filament as a function of time for the three ELM types. The type I and III ELM filaments have a radial acceleration of $\sim 2 \times 10^{8} \mathrm{~ms}^{-2}$ and an average radial velocity $\left(\mathrm{V}_{\mathrm{r}}\right)$ of $\sim 4000 \mathrm{~ms}^{-1}$ during the observed time interval. The small ELMs have a constant radial velocity of $2200 \mathrm{~ms}^{-1}$ which is much lower than the average radial velocity of type I and type III ELMs. Figure $9 \mathrm{~b}$ shows the probability distribution function of the average radial velocity for all the filaments observed, which confirms this trend of the filaments in small ELMs having approximately half of the average radial velocity associated with filaments in type I and type III ELMs.

This is consistent with the model for filament propagation presented in reference [17], which showed how the radial evolution of the filaments relates to the ratio of the density in the filament relative to the density at the target prior to the ELM. In particular, for filaments with high density and temperature the model predicts that the polarisation current flowing in the filaments can not be short-circuited through the divertor plates. This then leads to an acceleration of the filaments. In contrast, the polarization current for low density filaments, such as are normally observed in L-mode, can be short-circuited through the divertor plates and a constant radial velocity is predicted.

Rather than reporting individual filament motion the radial propagation of the filaments can also be captured in terms of a radial e-folding length of the density. Measurements of the peak value of the ion saturation current density $\left(\mathrm{J}_{\mathrm{SAT}}\right)$ as a function of distance from the LCFS ( $\left.\Delta \mathrm{R}_{\mathrm{LCFS}}\right)$ have been made using the Langmuir probe located on the reciprocating probe at the outboard mid-plane. The radial profiles obtained are shown in Figure 10 for type I and small ELMs. The size of the $\mathrm{J}_{\mathrm{SAT}}$ signals, at a given radial 
separation, is up to an order of magnitude smaller in the case of the small ELMs, consistent with the lower intensities observed in the visible imaging. The net radial flux of particles integrated over the time period of the ELM has been estimated using the measured values of $V_{r}$ from the image analysis presented above and the $J_{S A T}$ signal assuming a temperature of $20 \mathrm{eV}$ to be $6.8 \times 10^{18} \mathrm{~m}^{-2}$ for type I ELMs and $1.7 \times 10^{17} \mathrm{~m}^{-2}$ for type II ELMs.

The $\mathrm{J}_{\mathrm{SAT}}$ e-folding length $\left(\lambda_{\mathrm{JSAT}}\right)$ is similar in both cases with $\lambda_{\mathrm{JSAT}} \sim 35 \mathrm{~mm}$. In the simplest picture where the filaments propagate out radially with a constant velocity $\left(V_{r}\right)$ and lose particles on ion parallel transport timescales $\left(\tau_{/ /}=L_{/ /} / c_{s}, L_{/ /}\right.$is the connection length and $c_{s}$ the ion sound speed) then the particle e-folding length $(\lambda)$ can be expressed as $\lambda \sim$ $V_{r} \tau_{/ /} \sim V_{r} L_{/ / /} c_{s}$. As was shown in [18], when the relevant values of $V_{r}, L_{/ /}$and $\mathrm{c}_{\mathrm{s}}$ are inserted the value of $\lambda$ predicted by this expression is larger than that measured experimentally, however, the scaling predicted by this expression seems to hold. Hence, since $\mathrm{L}_{/ /}$is effectively constant in these discharges, then the findings above that $V_{r}$ is approximately a factor of 2 smaller in the case of the small ELMs would imply that in order to obtain the same $\lambda, c_{s}$ must also be a factor of 2 smaller. This would require lower temperatures in the filaments associated with the small ELMs. This could be either because they originate with a lower temperature or because they cool more quickly. It is possible to construct a more complex model including the cooling of the filament due to the sheath boundary. This gives the following radial evolution for the density as the filament propagates:

$$
n=n_{0}\left(1+\frac{(\gamma-3 / 2) c_{s}^{0}}{3 L_{/ /} v_{r}} r\right)^{\frac{-3}{\gamma-3 / 2}}
$$


where $\gamma$ is the sheath heat transfer coefficient and $\mathrm{c}_{\mathrm{s}}{ }^{0}$ is the sound speed at the birth location of the filament. Although the precise radial evolution is not exactly the same, the characteristic radial decay length remains proportional to $V_{r} L / / / C_{s}{ }^{0}$. Hence, the faster cooling is probably not the dominant mechanism in determining the fall-off length. Since the electron temperature pedestal profiles are similar just before a type I or small ELM on MAST (Figure 11) the scenario where the small ELM filaments originate from a region of lower temperature would be consistent with the picture discussed above, where these filaments originated from a location further down the pedestal i.e. nearer to the LCFS than is the case for type I ELM filaments.

\section{The spatial and temporal structure of type II ELMs on ASDEX Upgrade}

The divertor on ASDEX Upgrade is often in a detached state where ELMs appear as a dip in the $\mathrm{D}_{\alpha}$ intensity at the divertor rather than as a peak. Therefore, the time of an individual type I ELM is normally identified using either the electric current into a divertor tile, measured as a voltage at a shunt resistor embedded in the tile mounting, or by the increase in the tungsten emission at the divertor. Figure $12 \mathrm{a}$ shows a time trace of the tungsten line emission for a type I ELM-ing period of the discharge where clear peaks can be observed due to the ELMs with a frequency of $\sim 100 \mathrm{~Hz}$. Unfortunately neither the electric current nor the tungsten emission technique is sensitive enough to detect individual type II ELMs (Figure 12b). In order to unambiguously identify the time of the type II ELMs the $\mathrm{D}_{\alpha}$ intensity at the limiter, recorded by a fast framing camera has been used (Figure 12c). The peaks observed in this signal where found to be correlated with drops in the line averaged density measured by an edge interferometer system as well as peaks observed in the target 
heat fluxes measured with an IR system and hence consistent with being due to the type II ELM event. The ELM frequency derived using this technique is in the range from 500 to $1000 \mathrm{~Hz}$. The time of the peak in either the divertor tungsten emission (for type I ELMs) or the limiter signal (for type II ELMs) is used to define $t_{\mathrm{ELM}}$ relative to other plots.

Several methods have been used previously to determine the propagation of type I ELMs on ASDEX Upgrade and a comparison of these is given in reference [18]. In this paper one of these methods, based on the high heat flux probe head, which has been shown to be compatible with the other measurement techniques, has been used to compare the radial propagation of type I and II ELMs. The high heat flux probe head is attached to the mid-plane manipulator on ASDEX Upgrade, which is located at the low field side about 31 $\mathrm{cm}$ above the midplane. The head, which is made of graphite, consists of two columns each consisting of 5 Langmuir pins separated by $3 \mathrm{~mm}$ (see figure 8 in reference [18]). The tips are mounted in same plane as the surrounding shield, analogous to flush-mounted probes in the divertor. The probe surface is tilted $14^{\circ}$ with respect to the toroidal direction. For the discharges discussed here the probes are connected such that pins 3 and 5 measure the floating potential and pin 4 measures the ion saturation current (see figure 8 in reference [18]). Reciprocations of the probe were performed during the type I and II ELM-ing periods of repeat discharges. The ion saturation current density $\left(\mathrm{J}_{\mathrm{SAT}}\right)$ was measured as a function of distance from the LCFS $\left(\Delta \mathrm{R}_{\mathrm{LCFS}}\right)$ during in and out strokes. The probe was reciprocated to a point where $\Delta \mathrm{R}_{\mathrm{LCFS}} \sim 3 \mathrm{~cm}$ and held constant at this location for $300 \mathrm{~ms}$ in order to get the maximum statistics at the closest point of approach. 
Figure 13a and $d$ show the ion saturation current density $\left(\mathrm{J}_{\mathrm{SAT}}\right)$ recorded by pin 4 in a $\pm 300 \mu$ s interval centred on the ELM peak time ( $\mathrm{t}_{\text {ELM }}$ ) for a type I and II ELM respectively, while the probe is $3 \mathrm{~cm}$ from the LCFS. Peaks in the $\mathrm{J}_{\mathrm{SAT}}$ signal during an ELM have been shown previously to be correlated with the arrival of filaments at the probe [18] and the filaments that are analysed are the ones that appear as prominent peaks. In type I ELMs normally about 5-10 peaks are observed and they are typically located within $\pm 100 \mu \mathrm{s}$ of $t_{\mathrm{ELM}}$. There are typically one or two large peaks and several smaller ones. For the type II ELMs, many more peaks are observed in the $\mathrm{J}_{\mathrm{SAT}}$ signal and the peaks are extended over a longer time duration. There is also a larger background $\mathrm{J}_{\mathrm{SAT}}$ level both between the filaments during a type II ELM and during the inter ELM period between type II ELMs compared to type I ELMs.

The radial velocity of the filaments has been determined assuming that their motion is due to an $\vec{E} \times \vec{B}$ drift. The poloidal electric field $\left(E_{\vartheta}\right)$ has been estimated by measurements of the floating potential from the two poloidally separated probes (pins 3 and 5) as $E_{\vartheta}=\left(V_{f l, 3}-V_{f l, 5}\right) / d$ where $\mathrm{d}=6 \mathrm{~mm}$ and the radial velocity is then calculated as $V_{r}=E_{\vartheta} / B$. In reference [18] it was shown that a consistent approximation of the radial velocity can be established with this method as long as the $\mathrm{J}_{\mathrm{SAT}}$ probe used to detect the filament is located poloidally between the two floating potential probes. In this current paper the same probe configuration has been used.

Figure $13 \mathrm{~b}$ and e show the radial velocity calculated using this technique. Large values of $V_{r}$ are produced, with $V_{r}$ up to $3000 \mathrm{~ms}^{-1}$, with the largest signals being in the vicinity of the peaks in the $J_{S A T}$. Figure $13 \mathrm{c}$ and $\mathrm{f}$ shows the radial flux calculated from $V_{r}$ 
and the $J_{S A T}$ signal assuming a temperature of $20 \mathrm{eV}$. Over the time period shown the integrated flux $\int \Gamma_{i, j} d t$ during type I ELMs $\left(5.3 \times 10^{16} \mathrm{~m}^{-2}\right)$ is less than that during type II ELMs $\left(30.0 \times 10^{16} \mathrm{~m}^{-2}\right)$. Surprisingly, the net radial efflux of particles during a type II ELMing period is larger than that during type I ELMs (see also reference [19]). Some of the enhanced flux in the type II ELMs is due to the larger $\mathrm{J}_{\mathrm{SAT}}$ signals in between the filaments but the effect of this is estimated to be less than $10 \%$ of the total flux. This larger flux of particles results in a larger density in the Scrape Off Layer (SOL), which can be observed in the density profile shown in Figure 14a. The profiles shown in Figure 14 are obtained in the last $10 \%$ of the ELM cycle (i.e. just before the ELM crash) for the type I ELMs and are ELM averaged in the case of the type II ELMs. The electron temperature and density profiles come from a Thomson scattering system and the ion temperature and toroidal velocity are measured using a charge exchange recombination spectroscopy system. As can be seen from Figure 14 there is very little difference in the density profiles inside the LCFS for the two ELM types. During the type II ELMing period the electron temperature inside the pedestal is lower by $\sim 100 \mathrm{eV}$ compared to the type I ELMing period (Figure 14b), however, in the region from which the filaments are likely to originate i.e. between the pedestal and the LCFS, the two distributions are similar. There is also little difference in this region for either the ion temperature (Figure 14c) or the toroidal rotation velocity of the plasma (Figure 14d).

Following the method used in [18], a weighted smoothed radial velocity has been calculated as $V_{r}=\left\langle J_{S A T} E_{\vartheta}\right\rangle_{d t} /\left\langle J_{S A T}\right\rangle_{d t} B$ where the integration time chosen was $d t=5 \mu \mathrm{s}$. This was the value found in reference [18] to be the optimum one that would smooth out 
some of the largest fluctuations while producing measurements of the radial velocities compatible with other techniques. Figure 15 shows the probability distribution obtained applying this technique to 318 filaments observed in type I ELMs and 4968 filaments in type II ELMs. The mean value of the radial velocity $\left(\left\langle V_{r}\right\rangle\right)$ and standard deviation $(\sigma)$ obtained is: for type I ELMs $\left\langle V_{r}\right\rangle=1640 \mathrm{~ms}^{-1}, \sigma=916 \mathrm{~ms}^{-1}$ and for type II ELMs $\left\langle V_{r}>=780 \mathrm{~ms}^{-1}, \sigma=887 \mathrm{~ms}^{-1}\right.$. Hence similar to what was observed on MAST smaller ELMs have a lower radial velocity.

Measurements of the peak value of the ion saturation current density current $\left(\mathrm{J}_{\mathrm{SAT}}\right)$ as a function of distance from the LCFS ( $\left.\Delta \mathrm{R}_{\mathrm{LCFS}}\right)$ have also been made using the Langmuir probe located on the mid-plane manipulator. The radial profiles obtained are shown in Figure 16a and $\mathrm{b}$ for type I and II ELMs respectively. The size of the $\mathrm{J}_{\mathrm{SAT}}$ signals, at a given radial separation and the e-folding length $\left(\lambda_{\mathrm{JSAT}}\right)$ are very similar in the two cases. As discussed above, the $\mathrm{J}_{\mathrm{SAT}}$ signal between the peaks does not go to zero in the case of type II ELMs. If instead of plotting the peak $\mathrm{J}_{\mathrm{SAT}}$ value, the inter filament background was subtracted it would reduce the peak $\mathrm{J}_{\mathrm{SAT}}$ values in the type II ELMs by less than $10 \%$ while leaving the type I distribution effectively unchanged and would result in a negligible effect on the measured e-folding lengths.

The simple relationship where the characteristic radial decay length is proportional to $V_{r} L / / / c_{s}^{0}$, combined with the measurements of $V_{\mathrm{r}}$ above, would imply that $c_{s}^{0}$ must also be a factor of 2 smaller for type II ELMs. However, the temperature profiles in the region from the pedestal towards the LCFS (see Figure $14 \mathrm{~b}$ and Figure 14c) are similar which 
would require that the type II ELM filaments originate from a location further down the pedestal (i.e. nearer to the LCFS).

In order to try to determine the toroidal mode number of the ELMs, the number of peaks in the $\mathrm{J}_{\mathrm{SAT}}$ trace and their temporal separation has been determined in a $\pm 300 \mu \mathrm{s}$ interval relative to $\mathrm{t}_{\mathrm{ELM}}$ for 124 type I Elms and 1317 type II ELMs. The probability distribution (Figure 17a) shows that on average twice as many peaks are observed during a type II ELM compared to a type I ELM, however, the time separation between the peaks (not shown) is similar for both types. The toroidal mode number can be estimated from this time differences $(\Delta t)$, the toroidal rotation velocity of the filaments $\left(V_{\phi}\right)$ and the toroidal circumference around the outside of the plasma $\left(L_{\text {outer }} \sim 13.5 \mathrm{~m}\right)$ using mode number $=\frac{L_{\text {outer }}}{V_{\phi} \Delta t}$. The toroidal rotation velocity $\left(\mathrm{V}_{\phi}\right)$ of the filaments has been determined using the time delay between the peaks in the ion saturation current obtained from two pins on the mid-plane manipulator probe head that are at the same radial location but are separated in the toroidal direction by $9 \mathrm{~mm}$. The data acquisition rate of the $\mathrm{J}_{\mathrm{SAT}}$ signal means that the time delay can only be resolved to $0.5 \mu$ s resulting in a maximum $V_{\phi}$ that can be determined of $18 \mathrm{kms}^{-1}$. The distribution of the derived values of $\mathrm{V}_{\phi}$ are shown in Figure $17 \mathrm{~b}$. The filaments are observed to rotate in the same direction as the bulk plasma with type I ELMs rotating on average, twice as fast as type II ELMs. In both cases the filament velocity is smaller than that of the pedestal velocity which for both the type I and II periods of these discharges is $\sim 25 \mathrm{kms}^{-1}$ (Figure $14 \mathrm{~d}$ ). 
Previous measurements of the filaments in type I ELMs on ASDEX Upgrade using visible imaging [20] showed that the filaments start off rotating toroidally in the co-current direction with toroidal velocities in the range of the velocity of the pedestal $\sim 30 \mathrm{kms}^{-1}$. The images revealed that after some time the toroidal rotation of an individual filament slows and soon afterwards hits the limiter. Hence it would seem probable that somewhere between the filament separating from the plasma edge and arriving at the mid-plane probe, located $5 \mathrm{~cm}$ from the plasma edge the toroidal velocity would decrease. Assuming that this deceleration is similar for the both type I and II ELMs then this would suggest that the filaments associated with the type II ELMs originate from a different location in the pedestal (i.e. a location of slower rotation speed) compared to the type I ELMs. Using the measured toroidal rotation speeds and the temporal separation of the filaments the effective toroidal mode number of the ELM has been determined (Figure 17c). Type I ELMs have toroidal mode numbers in the range 5 to 30 with a mean of 15 while type II ELMs have mode numbers in the range of 10 to 40 with a mean of 27 . The upper limit on the toroidal velocities that can be determined, which particularly affects the type I ELM measurements (see Figure 13b), may mean that the upper end of range of mode numbers in the case of the type I ELMs is overestimated.

\section{Summary and Discussions}

The characteristics of small ELMs on MAST and type II ELMs on ASDEX Upgrade have been studied and compared with the characteristics of type I ELMs on both devices. On both devices these small ELM regimes are established when the magnetic configuration of the plasma is close to a connected double null with high triangularity and 
in a limited region of normalised pedestal pressure and collisionality. On both devices the transition from type I to type II ELMs is connected with a similar threshold value of the pedestal collisionality.

ELM types are often parameterised in terms of their ideal MHD stability properties, where the edge stability is described in terms of a current density $(j)$ versus normalised pressure gradient diagram $(\alpha)[1]$. Whilst in a wide range of devices stability calculations show that the pedestal profiles obtained just before a type I ELM are located close to the peeling-ballooning boundary, the trend is less clear for other ELM types. For example, type III ELMs on JET [9] and MAST [21] appear to be stable to both low-n peeling modes and high-n ballooning modes. For the case of type II ELMs on ASDEX Upgrade [8] and JET [9] stability analyses have shown, that the near double null configuration required to access type II ELMs, considerably expands the stable region in the upper right corner of the $\alpha-j$ diagram. This means that the pedestal profiles are now in a region dominated by high-n ballooning modes, which is consistent with the higher toroidal mode numbers that have been observed and described in this paper. Unfortunately, similar stability analyses performed on MAST indicate that the profiles obtained just before the small ELMs are far from both the peeling and ballooning boundaries and hence these analyses can not be used to clearly identify the ELM type. This may be due to inadequacies in the experimental measurements or in the stability model. Therefore an alternative approach was used, based on a study of the filament properties observed during the ELMs.

The temporal and spatial evolution of the filament structures observed during the small ELMs on MAST and type II ELMs on ASDEX Upgrade have been studied. On both 
devices the toroidal mode number of the small ELMs is approximately twice as large as that of type I ELMs. The effective radial velocity of the filaments is also smaller by a factor of two compared to type I ELMs. The toroidal rotation velocity of the filaments is smaller for the small ELMs suggesting that they may originate from a location further down the pedestal (i.e. nearer to the LCFS) than type I ELM filaments. If they did originate from nearer the LCFS they may be expected to be populated with particles having lower temperatures and hence giving rise to lower values of the sound speed $c_{s}$. This would then explain why the $\mathrm{J}_{\mathrm{SAT}}$ e-folding length for type I and small ELMs is the same in spite of the different radial velocities.

In summary the characteristics of small ELMs on MAST and type II ELMs on ASDEX Upgrade are so similar that there would be no justification in defining them as different types. The only real difference is that in MAST the filaments in the small ELMs have significantly smaller densities than those associated with type II ELMs on ASDEX Upgrade. In fact, the radial flux of particles during a type II ELM on ASDEX Upgrade is larger than a type I ELM. Although we have no explanation why the filaments on ASDEX Upgrade are more dense, the difference in radial efflux during these ELMs may explain why the type II ELM-ing period can be sustained on ASDEX Upgrade while being transient on MAST. The smaller flux on MAST means that the pedestal continues to evolve until a type I ELM is triggered whereas on ASDEX Upgrade the large flux of particles stabilises the pedestal and prevents a type I ELM being triggered. The fact that the same type of small ELM can be produced on two very dissimilar devices opens the possibility that there may be multiple ways to obtaining small ELM regimes. In addition, it raises the possibility that the grassy ELM regime, which has many similarities to type II ELMs may be 
accessible in a spherical tokomak. The advantage of the grassy ELM regime, which has been observed on JT60-U, JET and AUG, is that it is obtained at low collisionalities $\left(\mathrm{ve}^{*}<\right.$ 1) and hence is more relevant to future devices.

\section{Acknowledgement}

This work, part-funded by the European Communities under the contract of Association between EURATOM and CCFE, was carried out within the framework of the European Fusion Development Agreement. The views and opinions expressed herein do not necessarily reflect those of the European Commission. This work was also part-funded by the RCUK Energy Programme under grant EP/I501045. 


\section{References}

[1] Wilson H et al., 2006 Plasma Phys. Control. Fusion 48 A71

[2] Kamiya K et al., 2007Plasma Phys. Control. Fusion 49 S43

[3] Loarte A et al. 2002 Plasma Phys. Control. Fusion 441815

[4] Oyama N et al. 2006 Plasma Phys. Control. Fusion 48 A171

[5] Sauter O, Angioni C and Lin-Liu Y R 1999 Phys. Plasmas 62834

[6] Stober J. et al., 2001 Nucl. Fusion 411123

[7] Saibene G. et al., 2005 Nucl. Fusion 45297

[8] Saarelma S. et al., 2003 Nucl. Fusion 43262

[9] Saarelma S. et al., 2009 Plasma Physics and Contr. Fusion 51035001

[10] Perez von Thun C. et al., 2008 Plasma Phys. Control. Fusion 50065018

[1] Wolfrum E. et al., "Edge profile and MHD characterisation of the type-II ELMy regime in ASDEX Upgrade" Europhysics Conference Abstracts (CD-ROM, Proc. of the 37th EPS Conference on Plasma Physics, Dublin, Ireland, 2010), (Ed.) C. McKenna (European Physical Society, Geneva), Vol. 34A (2010), P2.169 and submitted to Plasma Phys. Control. Fusion.

[12] Meyer H. et al., 2009 Nucl. Fusion 49104017

[13] Maingi R et al., 2011 Nucl. Fusion 51063036

[14] Kirk A et al. 2009 J. Nucl. Mater. 390-391 727

[15] Kirk A et al. 2006 Phys. Rev. Lett. 96185001

[16] Ben Ayed N. et al., 2009 Plasma Physics and Contr. Fusion 51035016

[17] Rozhansky V, 2008 Plasma Phys. Control. Fusion 50025008 
[18] Kirk A et al. 2011 Plasma Phys. Control. Fusion 53035003

[19] Mueller H.W. et al., "Fluctuations, ELM Filaments and Turbulent Transport in the SOL at the Outer Midplane of ASDEX Upgrade" Proc of 23rd IAEA Fusion Energy Conf, (Daejeon) EXX/P3-23. Submitted to Nucl. Fusion.

[20] Kirk A et al. 2008 J. Phys.: Conf. Ser. 123012012

[21] Kirk A et al. 2009 Plasma Phys. Control. Fusion 51065016 
Figures

Figure 1 Time traces of the mid-plane $\mathrm{D}_{\alpha}$ and the distance between the two separatrices at the Low Field Side mid-plane on ASDEX Upgrade for a) shot 25724 with a transient type II ELM period from 3.2 to $3.5 \mathrm{~s}$ and b) shot 25727 with a sustained type II ELM period after $2.9 \mathrm{~s}$.

Figure 2 Time traces of the mid-plane $\mathrm{D}_{\alpha}$ on MAST for shots with injected neutral beam powers $\left(\mathrm{P}_{\mathrm{NBI}}\right)$ of a) $\left.1.3, \mathrm{~b}\right) 1.5$ and c) $1.7 \mathrm{MW}$.

Figure 3 Existence space of normalised pedestal pressure $\left(\beta_{\text {ped }}\right)$ versus pedestal collisionality $\left(v^{*}\right)$ as a function of ELM type for a) MAST and b) ASDEX Upgrade.

Figure 4 a) $D_{\alpha}$ time trace during small ELMs on MAST. The vertical line shows the time of the visible image shown in b) which is a view of a mid-plane portion of the plasma, showing a large number of discrete filaments.

Figure 5 Time traces of a) $\mathrm{D}_{\alpha}$ and $\mathrm{b}$ ) ion saturation current $\left(\mathrm{I}_{\mathrm{SAT}}\right)$ recorded by a Langmuir probe located $5 \mathrm{~cm}$ from the last closed flux surface at the low field side mid-plane. The vertical lines show the location in time of the visible images shown at the bottom.

Figure 6 Average image intensity as a function of toroidal angle for the image shown in frame 2 of Figure 5. For each filament the toroidal location (vertical line) and the half width half maximum toroidal extent (horizontal line) determined are shown.

Figure 7 Time traces of a) $D_{\alpha}$ and b) the toroidal location of each filament during a small ELM on MAST.

Figure 8 Probability distribution of a) the toroidal velocity of the filaments $\left(\mathrm{V}_{\phi}\right)$ and $b$ ) toroidal mode number as a function of ELM type on MAST.

Figure 9 a) Radial position of a filament relative to the last closed flux surface $\left(\Delta \mathrm{R}_{\mathrm{LCFS}}\right)$ as a function of time in the ELM and b) the probability distribution of the derived radial velocity $\left(\mathrm{V}_{\mathrm{r}}\right)$ for different ELM types on MAST.

Figure 10 Ion saturation current density $\left(\mathrm{J}_{\mathrm{SAT}}\right)$ as a function of distance from the last closed flux surface $\left(\Delta \mathrm{R}_{\mathrm{LCFS}}\right)$ for type I and small ELMs on MAST.

Figure 11 Electron temperature as a function of distance from the last closed flux surface $\left(\triangle \mathrm{R}_{\mathrm{LCFS}}\right)$ obtained just before a type I and small ELM on MAST. 
Figure 12 Time traces of divertor $\mathrm{W}$ emission for periods of a) type I and $\mathrm{b}$ ) type II ELMs from shot 25727 on ASDEX Upgrade. c) Time trace of the $D_{\alpha}$ intensity from the limiter region during a type II ELM period.

Figure 13 a) The ion saturation current $\left(\mathrm{J}_{\mathrm{SAT}}\right)$, b) the radial velocity $\left(\mathrm{E}_{\theta} \times \mathrm{B} / \mathrm{B}^{2}\right)$ and $\left.\mathrm{c}\right)$ the particle flux $(\Gamma)$ as function of time during a type I ELM from shot 25730 at $2.291 \mathrm{~s}$ in ASDEX Upgrade. d), e) and f) similar traces during a type II ELM from shot 25730 at $3.123 \mathrm{~s}$.

Figure 14 Profiles of a) electron density, b) electron temperature, c) ion temperature and d) plasma toroidal velocity in normalised flux space for type I and type II ELMs from shot 25728 on ASDEX Upgrade.

Figure 15 The probability density function of the time averaged ion saturation current weighted radial velocity $\left(\mathrm{E}_{\theta} \times \mathrm{B} / \mathrm{B}^{2}\right)$ time averaging over $5 \mu$ s for type I (solid) and type II (dashed) ELMs.

Figure 16 Ion saturation current density $\left(\mathrm{J}_{\mathrm{SAT}}\right)$ as a function of distance from the last closed flux surface $\left(\Delta \mathrm{R}_{\mathrm{LCFS}}\right)$ for a) type I and $\mathrm{b}$ ) type II ELMs on ASDEX Upgrade.

Figure 17 Probability distributions of a) number of peaks per ELM in the ion saturation current distribution, b) toroidal velocity of the ELM filaments and c) toroidal mode number for type I (solid) and type II (dashed) ELMs in ASDEX Upgrade. 

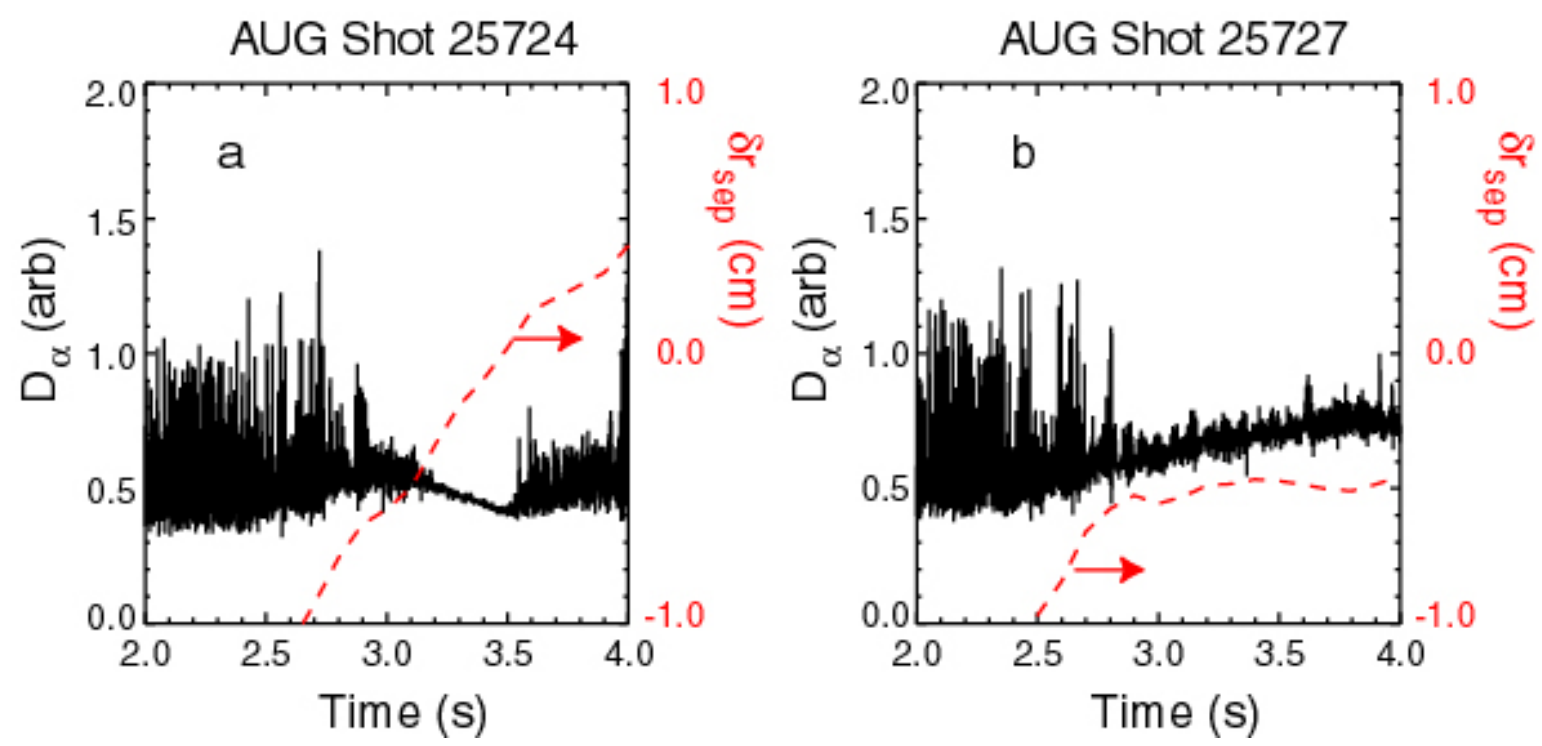

Figure 1 Time traces of the mid-plane $\mathrm{D}_{\alpha}$ and the distance between the two separatrices at the Low Field Side mid-plane on ASDEX Upgrade for a) shot 25724 with a transient type II ELM period from 3.2 to $3.5 \mathrm{~s}$ and b) shot 25727 with a sustained type II ELM period after $2.9 \mathrm{~s}$.

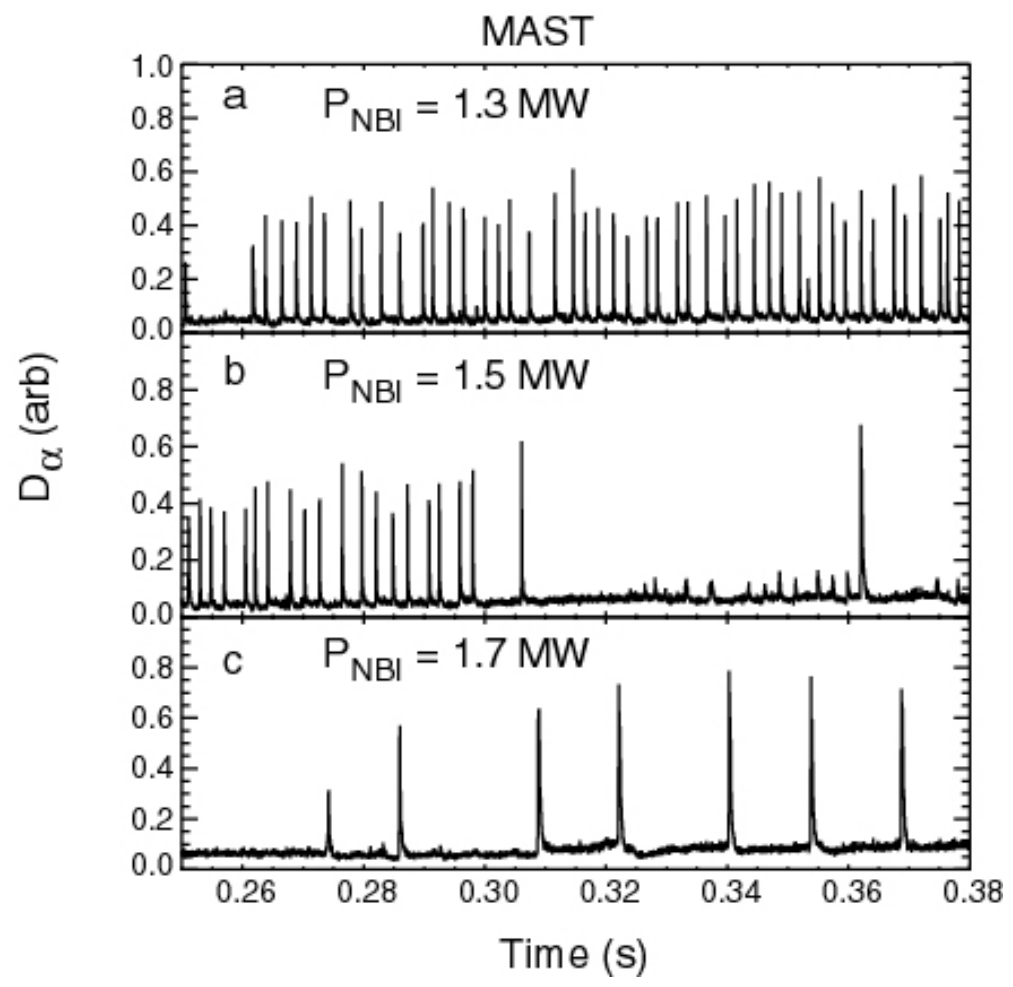

Figure 2 Time traces of the mid-plane $\mathrm{D}_{\alpha}$ on MAST for shots with injected neutral beam powers $\left(\mathrm{P}_{\mathrm{NBI}}\right)$ of a) 1.3 , b) 1.5 and c) $1.7 \mathrm{MW}$. 

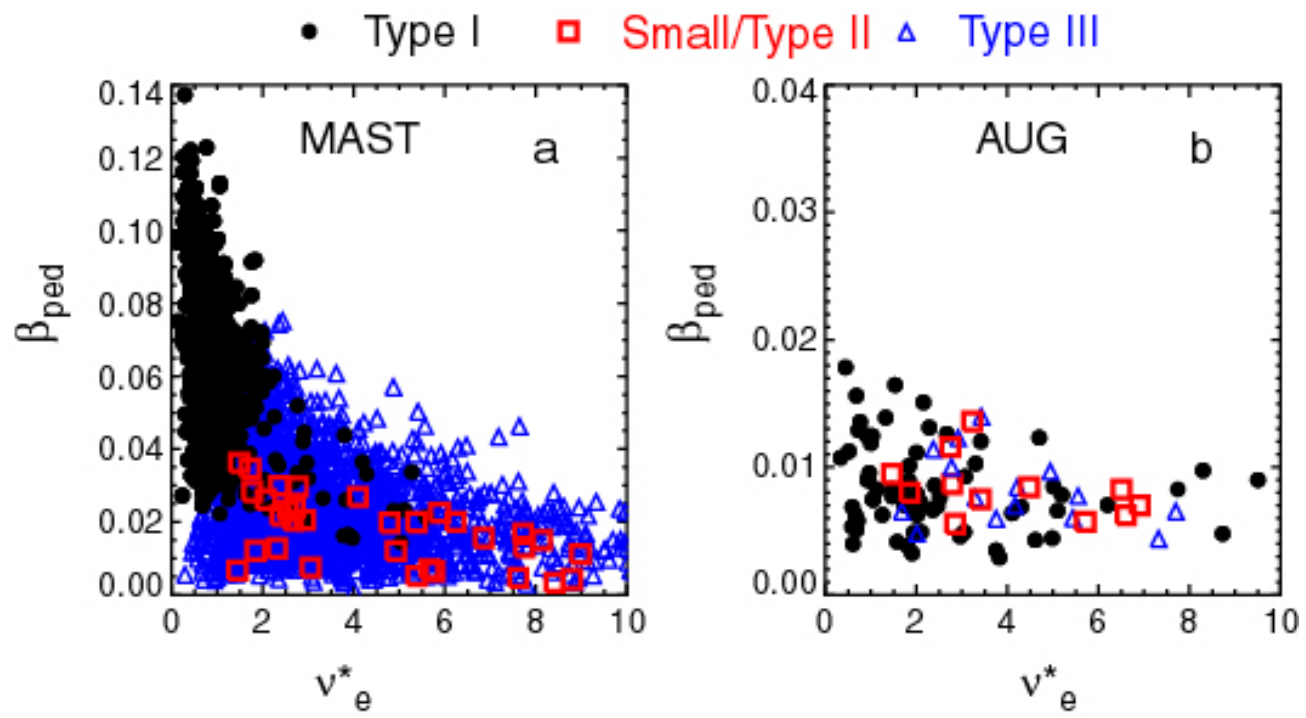

Figure 3 Existence space of normalised pedestal pressure $\left(\beta_{\text {ped }}\right)$ versus pedestal collisionality $\left(v_{e}^{*}\right)$ as a function of ELM type for a) MAST and b) ASDEX Upgrade.

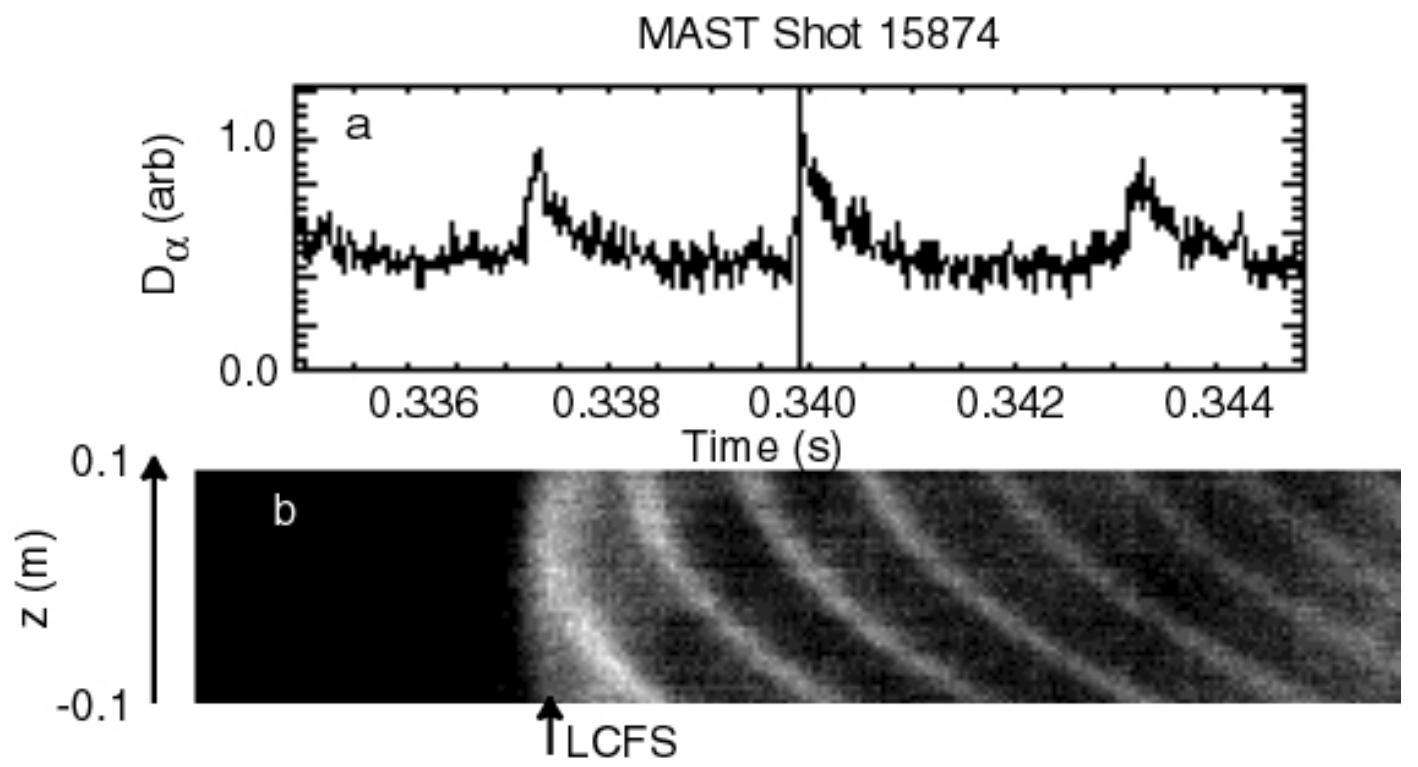

Figure 4 a) $D_{\alpha}$ time trace during small ELMs on MAST. The vertical line shows the time of the visible image shown in b) which is a view of a mid-plane portion of the plasma, showing a large number of discrete filaments. 

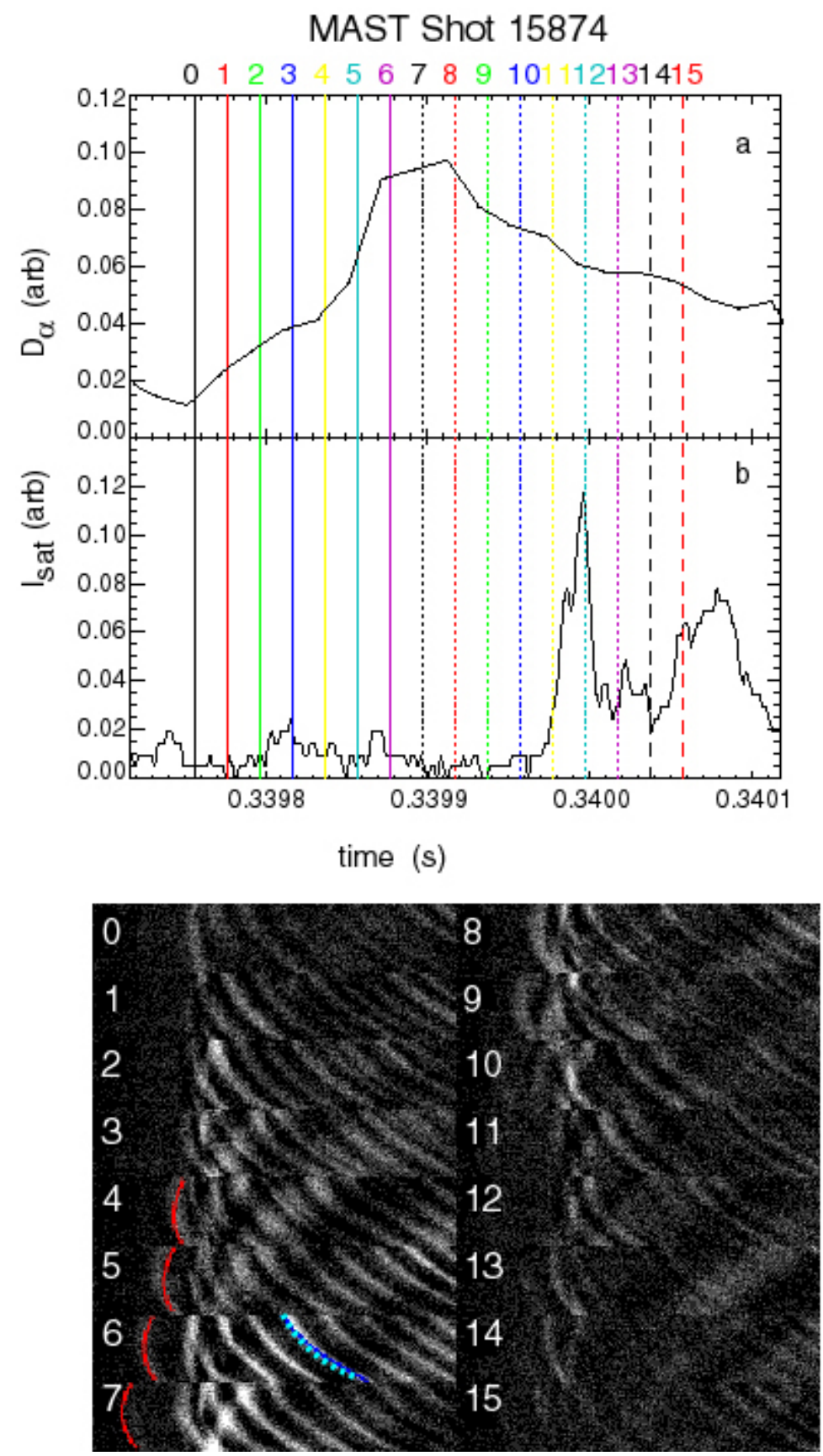

Figure 5 Time traces of a) $\mathrm{D}_{\alpha}$ and $\mathrm{b}$ ) ion saturation current $\left(\mathrm{I}_{\mathrm{SAT}}\right)$ recorded by a Langmuir probe located $5 \mathrm{~cm}$ from the last closed flux surface at the low field side mid-plane during a small ELM on MAST. The vertical lines show the location in time of the visible images shown at the bottom. The dotted blue curve in frame 6 represents a filament that is located at the LCFS, whilst the solid red curve in frames 4 to 7 represents a filament that is propagating radially outwards. 


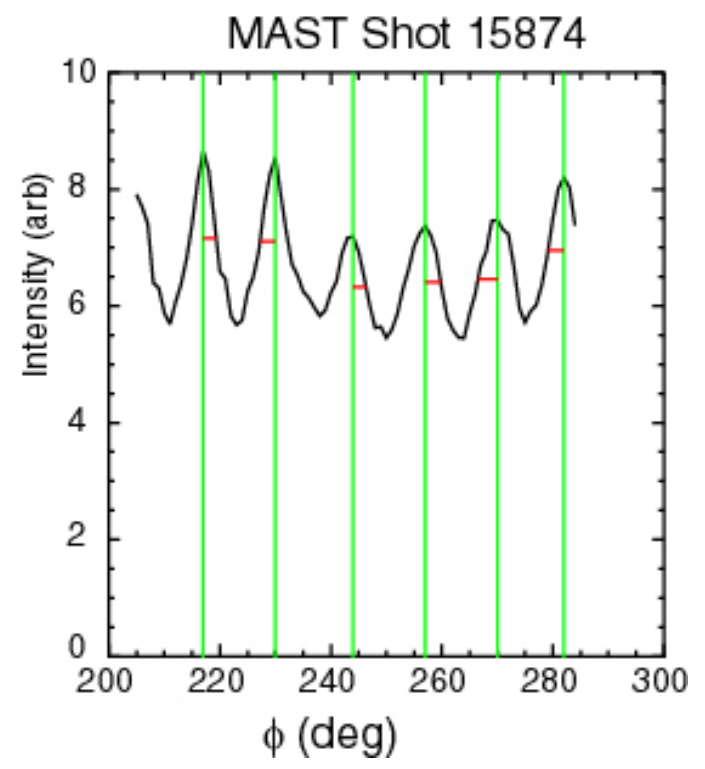

Figure 6 Average image intensity as a function of toroidal angle for the image shown in frame 2 of Figure 5. For each filament the toroidal location (vertical line) and the half width half maximum toroidal extent (horizontal line) determined are shown. 


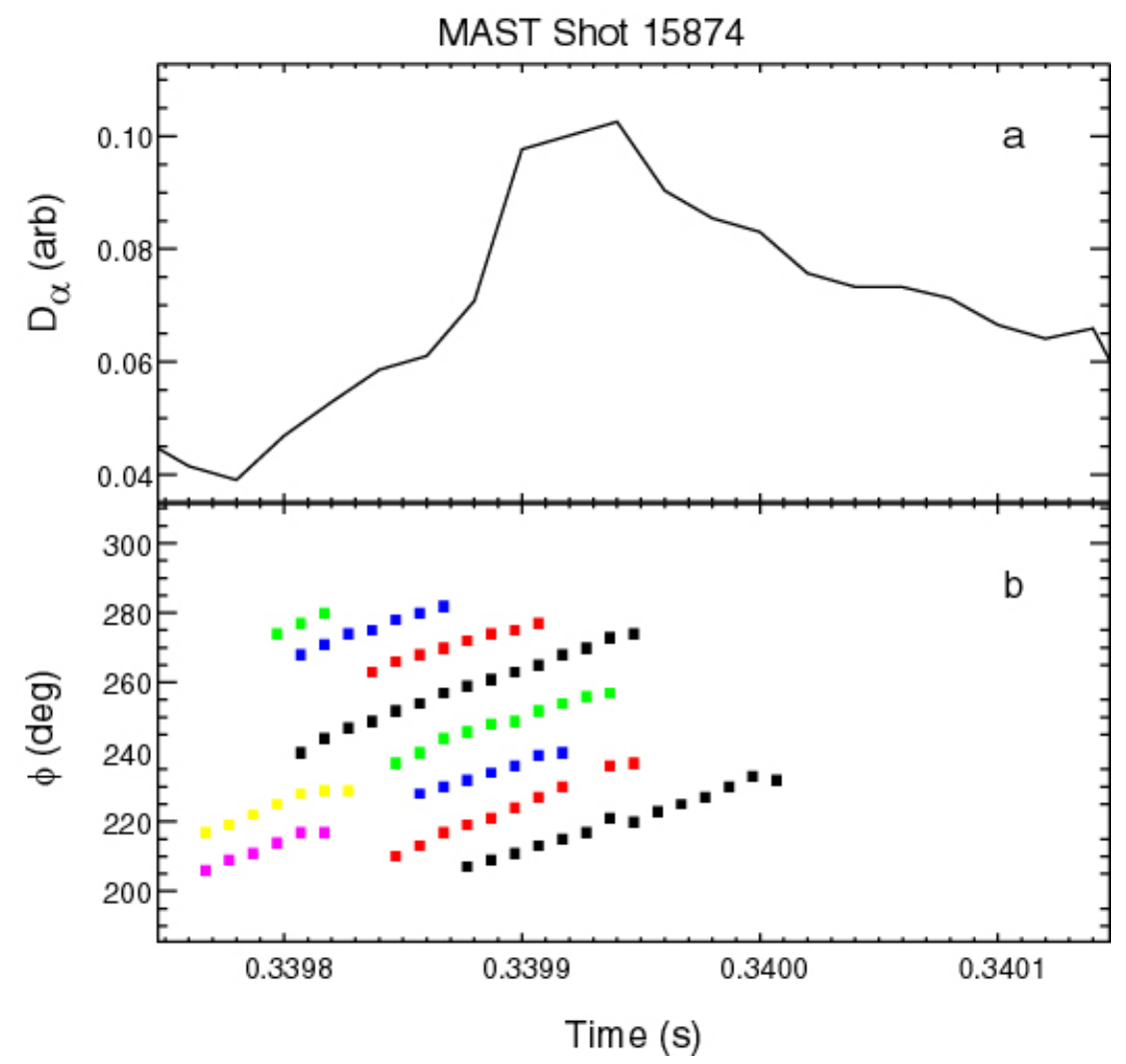

Figure 7 Time traces of a) $D_{\alpha}$ and b) the toroidal location of each filament during a small ELM on MAST. 

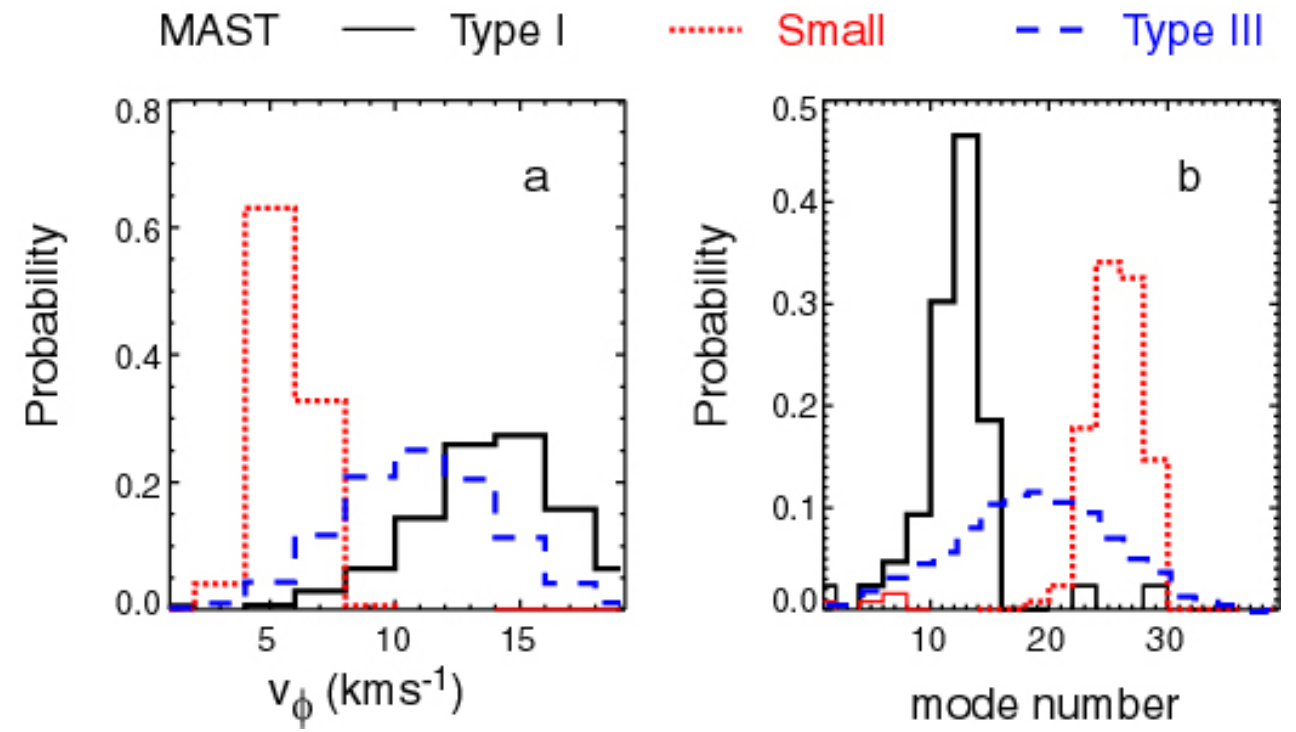

Figure 8 Probability distribution of a) the toroidal velocity of the filaments $\left(\mathrm{V}_{\phi}\right)$ and $b$ ) toroidal mode number as a function of ELM type on MAST.
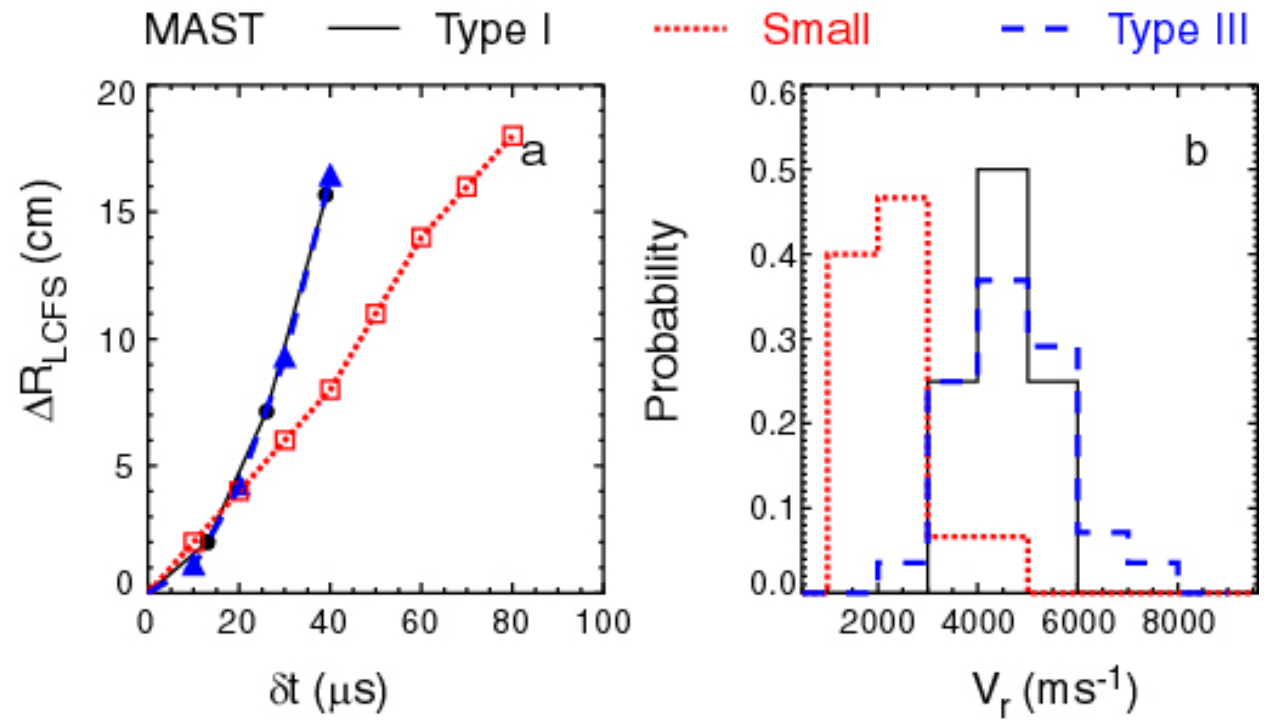

Figure 9 a) Radial position of a filament relative to the last closed flux surface $\left(\Delta R_{L C F S}\right)$ as a function of time in the ELM and $b$ ) the probability distribution of the derived radial velocity $\left(V_{r}\right)$ for different ELM types on MAST. 


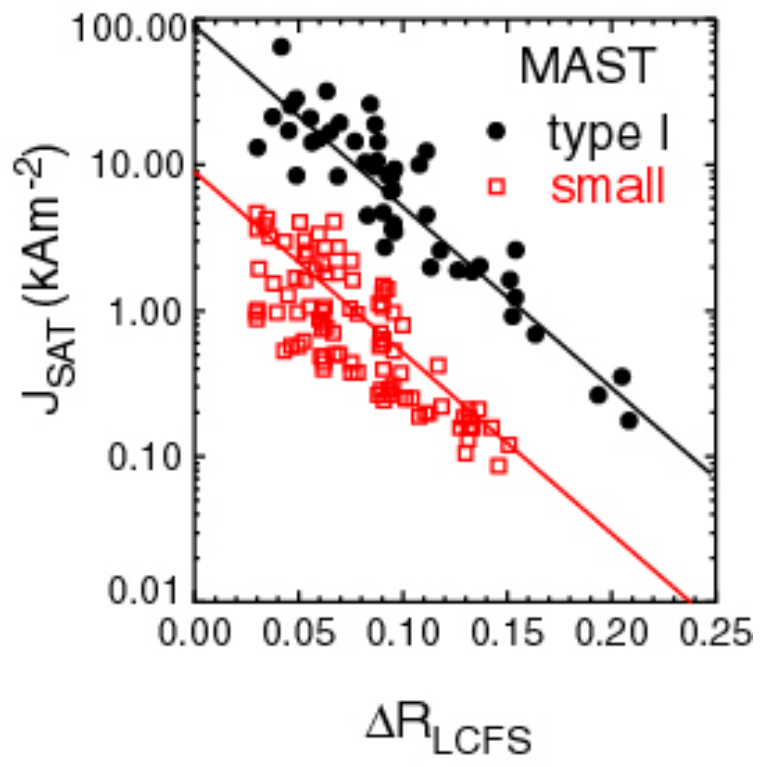

Figure 10 Ion saturation current density $\left(\mathrm{J}_{\mathrm{SAT}}\right)$ as a function of distance from the last closed flux surface $\left(\Delta \mathrm{R}_{\mathrm{LCFS}}\right)$ for type I and small ELMs on MAST.

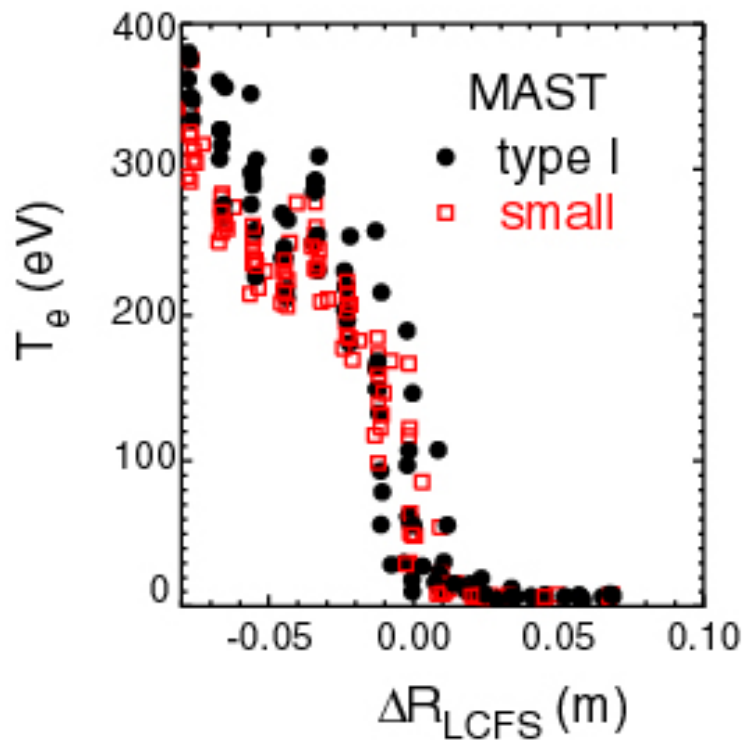

Figure 11 Electron temperature as a function of distance from the last closed flux surface $\left(\Delta \mathrm{R}_{\mathrm{LCFS}}\right)$ for obtained just before a type I and small ELM on MAST. 

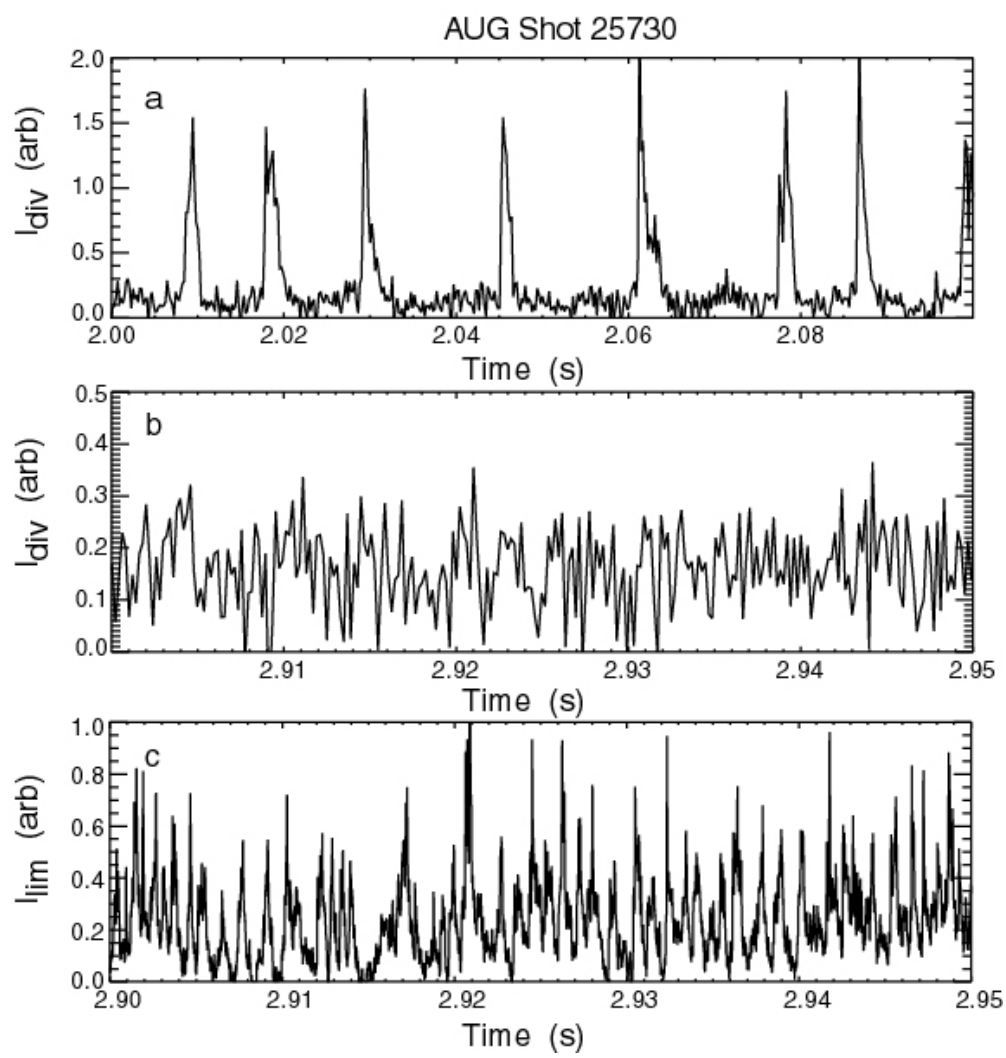

Figure 12 Time traces of divertor $\mathrm{W}$ emission for periods of a) type I and b) type II ELMs from shot 25727 on ASDEX Upgrade. c) Time trace of the $\mathrm{D}_{\alpha}$ intensity from the limiter region during a type II ELM period. 

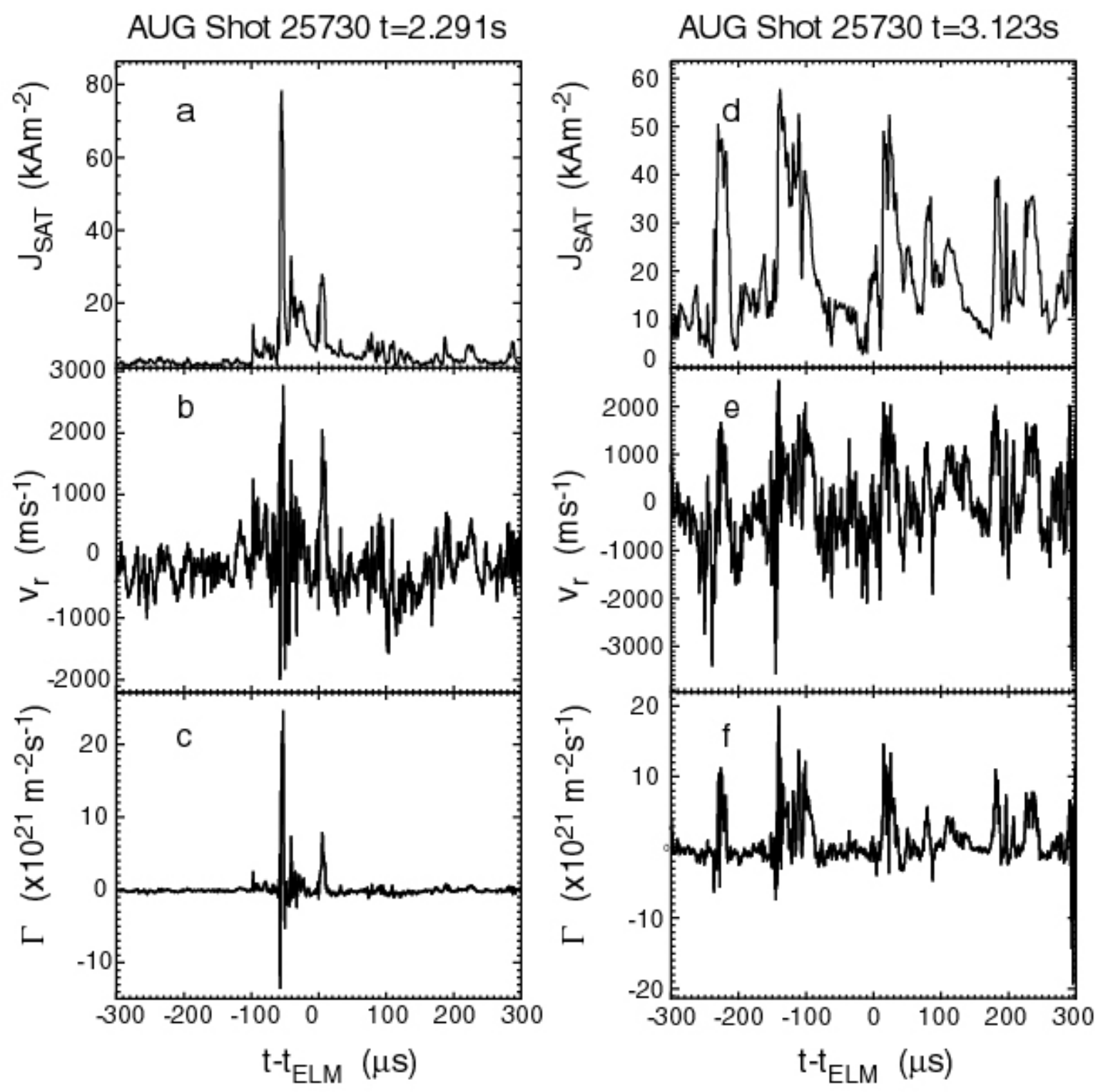

Figure 13 a) The ion saturation current $\left(\mathrm{J}_{\mathrm{SAT}}\right)$, b) the radial velocity $\left(\mathrm{E}_{\theta} \times \mathrm{B} / \mathrm{B}^{2}\right)$ and $\left.\mathrm{c}\right)$ the particle flux $(\Gamma)$ as function of time during a type I ELM from shot 25730 at $2.291 \mathrm{~s}$ in ASDEX Upgrade. d), e) and f) similar traces during a type II ELM from shot 25730 at $3.123 \mathrm{~s}$. 

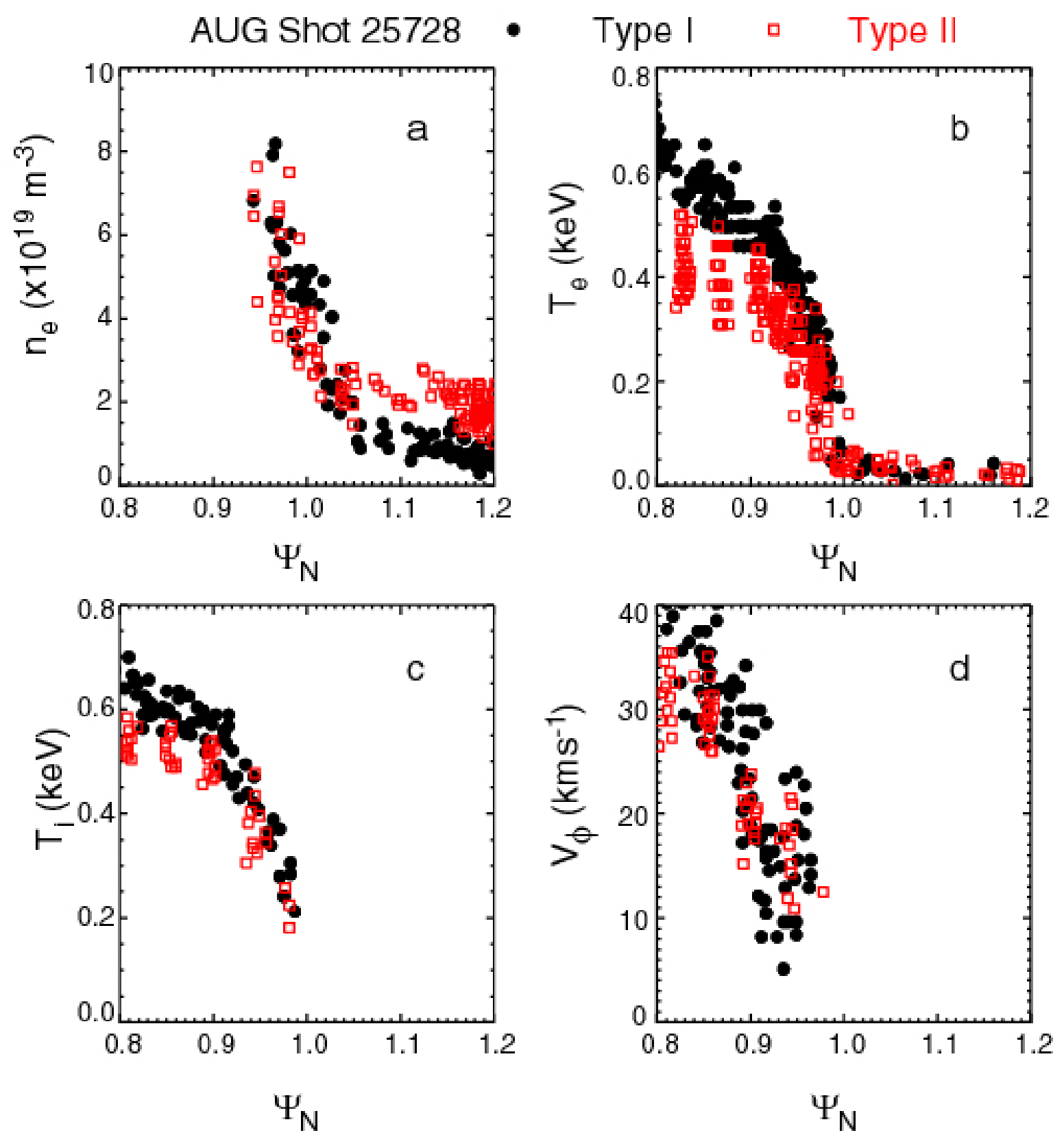

Figure 14 Profiles of a) electron density, b) electron temperature, c) ion temperature and d) plasma toroidal velocity in normalised flux space for type I and type II ELMs from shot 25728 on ASDEX Upgrade. 


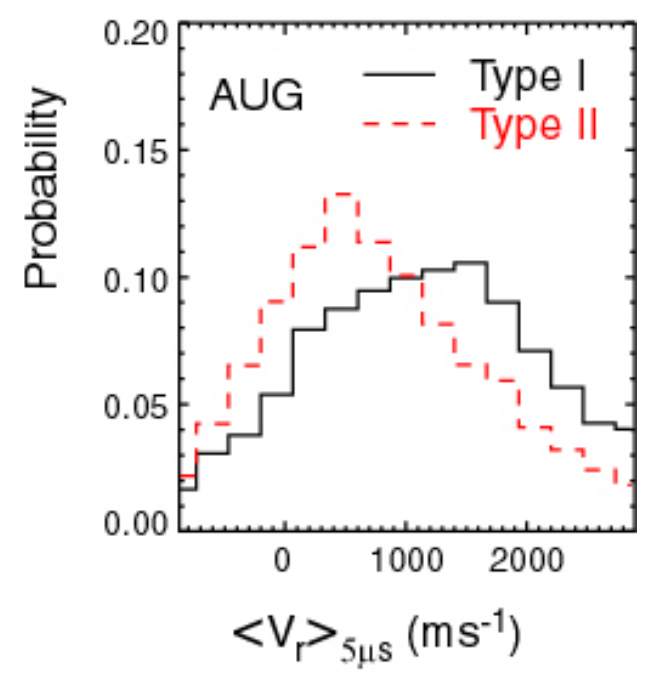

Figure 15 The probability density function of the time averaged ion saturation current weighted radial velocity $\left(\mathrm{E}_{\theta} \times \mathrm{B} / \mathrm{B}^{2}\right)$ time averaging over $5 \mu$ s for type I (solid) and type II (dashed) ELMs.
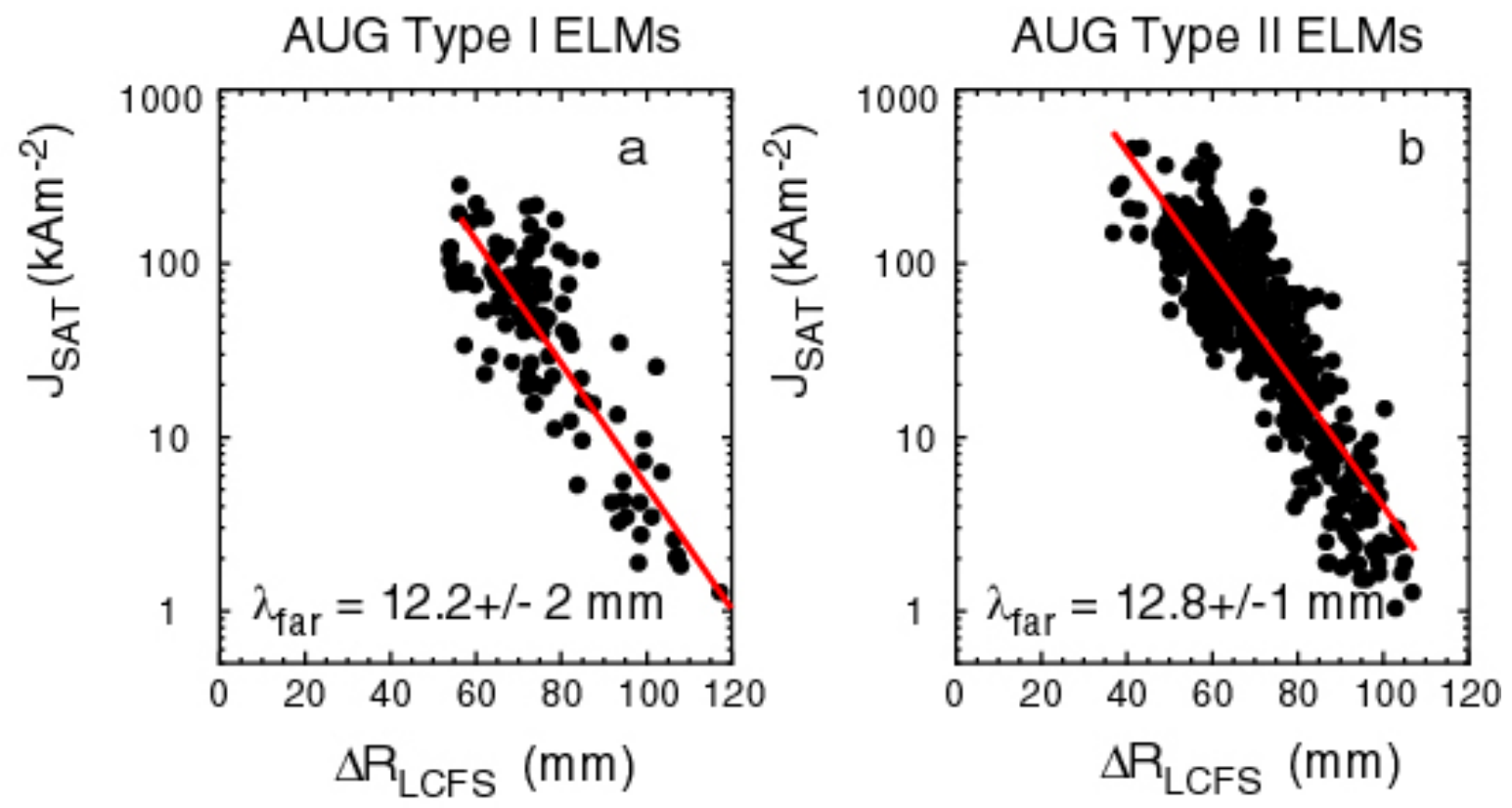

Figure 16 Ion saturation current density $\left(\mathrm{J}_{\mathrm{SAT}}\right)$ as a function of distance from the last closed flux surface $\left(\Delta \mathrm{R}_{\mathrm{LCFS}}\right)$ for a) type I and $\mathrm{b}$ ) type II ELMs on ASDEX Upgrade. 

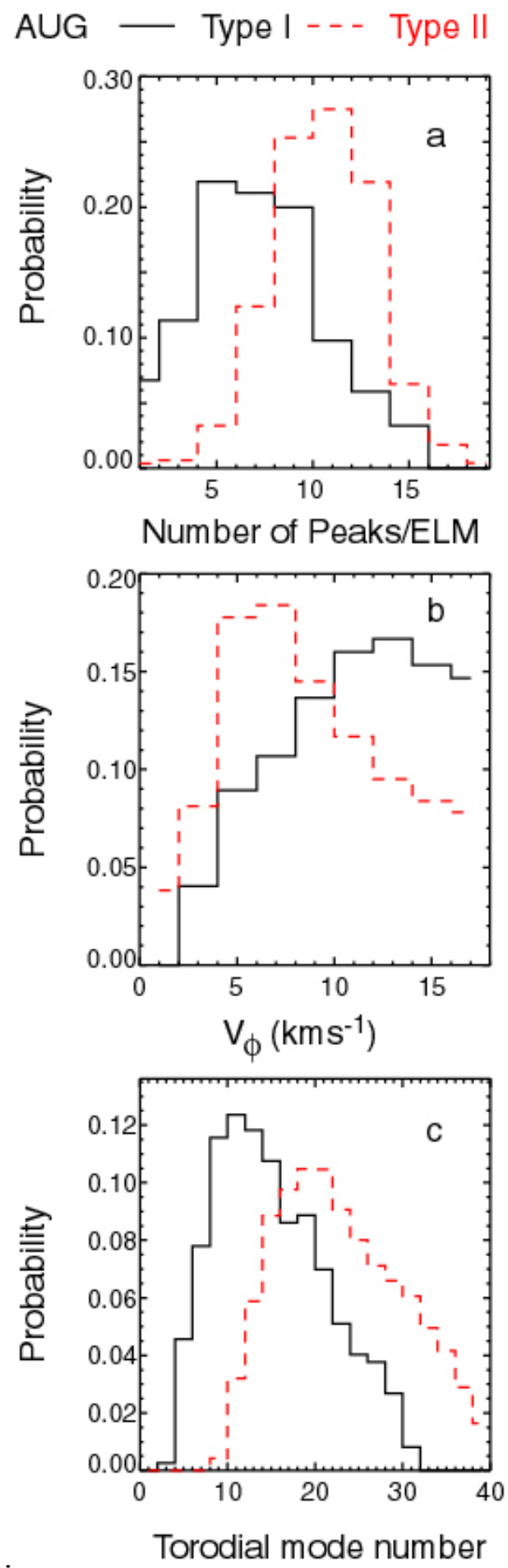

Figure 17 Probability distributions of a) number of peaks per ELM in the ion saturation current distribution, b) toroidal velocity of the ELM filaments and c) toroidal mode number for type I (solid) and type II (dashed) ELMs in ASDEX Upgrade. 